\title{
COMPARAÇÃO DE RESULTADOS OBSTÉTRICOS E NEONATAIS ENTRE PRIMÍPARAS E MULTÍPARAS ASSISTIDAS NO DOMICÍLIO
}

\author{
COMPARISON OF OBSTETRIC AND NEONATAL OUTCOMES BETWEEN \\ PRIMIPAROUS AND MULTIPAROUS ASSISTED AT HOME
}

\section{COMPARACIÓN DE LOS RESULTADOS OBSTÉTRICOS Y NEONATALES ENTRE PRIMÍPARAS Y MULTÍPARAS ASISTIDAS EN DOMICILIO}

\author{
Joyce Green KoetTKeR* \\ Odaléa Maria BrÜGgemann ${ }^{* *}$ \\ Rozany Mucha Dufloth ${ }^{* * *}$ \\ Marisa Monticelli ${ }^{* * *}$ \\ ROXANA KNOBEL ${ }^{* * * *}$
}

\begin{abstract}
RESUMO
Objetivo: Comparar os resultados obstétricos e neonatais entre primíparas e multíparas assistidas no domicilio por enfermeiras obstétricas. Material e método: Estudo de corte transversal, que analisou prontuários de 100 partos de mulheres assistidas no domicílio e seus neonatos, de janeiro/2005 a dezembro/2009, em Santa Catarina, Brasil. Os dados coletados dos prontuários foram analisados por estatística descritiva e inferencial. Resultados: 73,0\% das mulheres eram primíparas e 27,0\% eram multíparas. Houve diferença estatisticamente significativa quanto ao tempo transcorrido entre a primeira avaliação e o parto, que foi menor nas multíparas $(\mathrm{p}=0,0402)$. O banho terapêutico de aspersão foi o método mais utilizado para o alívio da dor pelas primíparas $(\mathrm{p}=0,0015)$, assim como a escolha pelo parto vertical na água $(\mathrm{p}=0,0004)$. Não houve diferença estatisticamente significativa para as demais variáveis obstétricas e neonatais e na taxa de transferência para o hospital. Conclusão: Diferente de outros países, a maioria das mulheres assistidas no domicílio era primípara. O estudo indica bons resultados maternos e neonatais, tanto em primíparas quanto em multíparas, e são semelhantes aos de outros países.
\end{abstract}

Palavras chave: Parto domiciliar, parto humanizado, enfermagem obstétrica, parto normal.

\footnotetext{
* Enfermeira obstétrica da Equipe Hanami - o florescer da vida - parto domiciliar planejado. Mestre em Enfermagem pela Universidade Federal de Santa Catarina (UFSC). Doutoranda em Enfermagem pela UFSC. Bolsista CAPES. Membro do Grupo de Pesquisa na Saúde da Mulher e do Recém-nascido (GRUPESMUR). Florianópolis, Santa Catarina, Brasil. E-mail: joycegreenk@yahoo.com.br. Endereço: Rua Rui Barbosa, 650 apto 801 torre 1, Agronômica, Florianópolis, SC, CEP. 88.025 $-301$.

** Doutora em Tocoginecologia. Docente do Departamento de Enfermagem e do Programa de Pós-Graduação em Enfermagem UFSC. Pesquisadora CNPq. Vice-líder do GRUPESMUR. Florianópolis, Santa Catarina, Brasil. E-mail: odalea@ccs.ufsc.br

*** Doutora em Tocoginecologia. Departamento de Patologia do Hospital do Câncer de Barretos, Fundação Pio XII e Faculdade de Ciências da Saúde de Barretos Dr. Paulo Prata (FASCIB) - Barretos, São Paulo, Brasil. E-mail: rozany.dufloth@ gmail.com

${ }_{* * * *}$ Doutora em Enfermagem. Docente aposentada do Departamento de Enfermagem e do Programa de Pós-Graduação em Enfermagem da UFSC. Florianópolis, Santa Catarina, Brasil. E-mail: marisa@ccs.ufsc.br

${ }^{* * * * *}$ Pós-doutora em Enfermagem. Docente do Departamento de Ginecologia e Obstetrícia da Universidade Federal de Santa Catarina. Florianópolis, Santa Catarina, Brasil. E-mail: knobel@uol.com.br
} 


\section{ABSTRACT}

Objective: To compare the obstetric and neonatal outcomes between primiparous and multiparous attended at home by obstetric nurses. Method: Cross sectional study, which examined 100 women attended births at home and their newborns from January 2005/December 2009, Brazil. The data collected from medical records were analyzed by descriptive and inferential statistics. Results: $73.0 \%$ of women were primiparous. There was statistically significant difference as to the time elapsed between the initial evaluation and delivery, which was smaller in multiparous ( $\mathrm{p}=0.0402$ ). The therapeutic bath of aspersion was the predominant method for pain relief by primiparous $(\mathrm{p}=0.0015)$, as well as the choice for vertical childbirth in water $(\mathrm{p}=0.0004)$. There was no statistically significant difference for the other obstetric and neonatal variables and in the transfer rate to hospital. Discussion and Conclusion: Different from other countries, the majority of women were primiparous. The results show good maternal and neonatal outcomes and are similar to those of other countries.

Key words: Home childbirth, humanizing delivery, obstetric nursing, natural childbirth.

\section{RESUMEN}

Objetivo: Comparar los resultados obstétricos y neonatales entre primíparas y multíparas atendidas en domicilio por enfermeras obstétricas. Material y método: Estudio de corte transversal, que examinó prontuarios de 100 mujeres que tuvieron sus partos en el hogar y sus recién nacidos de enero/2005 - diciembre/2009, Brasil. Los datos obtenidos de los registros médicos fueron analizados por estadística descriptiva e inferencial. Resultados: 73,0\% de las mujeres eran primíparas. Hubo diferencia estadísticamente significativa en cuanto al tiempo transcurrido entre la evaluación inicial y el parto, que fue menor en multíparas $(p=0,0402)$. El baño terapéutico de aspersión fue el método predominante para el alivio del dolor por primíparas ( $\mathrm{p}=0,0015)$, así como la elección para el parto vertical en agua $(\mathrm{p}=0,0004)$. No hubo diferencias estadísticamente significativas para las otras variables obstétricas y neonatales y en la tasa de transferencia al hospital. Conclusión: A diferencia de otros países, la mayoría de las mujeres eran primíparas. Los resultados muestran buenos resultados maternos y neonatales tanto en primíparas como en multíparas y son similares a los de otros países.

Palabras clave: Parto domiciliario, parto humanizado, enfermería obstétrica, parto normal.

Fecha recepción: 01/10/14 Fecha aceptación: 09/06/15

\section{INTRODUÇÃO}

O respeito à escolha da mulher sobre o local do parto, após ter recebido as informações necessárias, é uma das práticas recomendadas pela Organização Mundial da Saúde (OMS), podendo ser o domicílio, quando existir um centro de referência para transferência (1).

A OMS reconhece como profissional habilitado para prestar assistência ao parto: a enfermeira obstétrica, a obstetriz e o médico (1). No Brasil a enfermeira obstétrica é uma profissional habilitada e amparada legalmente pela Lei do Exercício para a assistência ao parto (2). Inclusive, em 2008 foi reconhecida pela Agência Nacional de Saúde Complementar para assistir ao parto domiciliar das gestantes de risco, de forma autônoma (3).

O Colégio Americano de Ginecologia e Obstetrícia não recomenda o parto domiciliar, independente da categoria profissional que o assiste, por apresentar risco de morte neonatal aumentado, em relação ao hospitalar, mesmo apontando menores índices de intervenções (4). No entanto, o atendimento 
ao parto domiciliar planejado vem crescendo mundialmente (5) e os resultados maternos e neonatais, de morbidade e mortalidade, têm sido amplamente publicados em periódicos internacionais. A maioria dos estudos, ao comparar a assistência no domicílio e no hospital, concluem que ambos os locais são igualmente seguros (6-13).

No Brasil, algumas mulheres estão optando pelo parto domiciliar, motivadas por algumas razões como: atendimento individualizado; pouca ou nenhuma intervenção obstétrica; contato pele a pele precoce e prolongado com o Recém-nascido (RN); e a possibilidade de ter um ou mais acompanhantes (14-16). Além disso, as elevadas taxas de cesárea $(3,17)$ e as experiências negativas acerca do parto têm influenciado as mulheres a desejarem esse tipo de atendimento já na primeira gestação.

Estudo multicêntrico realizado nas maternidades brasileiras apontou que somente $5,6 \%$ dos partos vaginais de mulheres de risco obstétrico habitual foram assistidos sem intervenções obstétricas (uso de cateter venoso, ocitocina para acelerar o trabalho de parto, amniotomia, analgesia raque/epidural, posição de litotomia, manobra de Kristeller e episiotomia) (17). Frequentes relatos de violência obstétrica no ambiente hospitalar, aliados a uma rede de informação sobre a possibilidade de que a mulher pode ter um parto ativo e humanizado no domicilio, oriundas de grupos de gestantes/casais grávidos, grupos virtuais e sites de informação, podem influenciar as mulheres a optar pela assistência nesse local (15). Uma pesquisa de opinião pública, realizada no Brasil, constatou violência sofrida no parto em $25,0 \%$ das entrevistadas que foram atendidas na rede pública ou privada (62\%), sendo $26,0 \%$ delas na Região Sul no Brasil. A violência verbal dos profissionais de saúde, relatada pelas mulheres, pode ser exemplificada por frases do tipo: "...não chora que ano que vem você está aqui de novo..." e “...na hora de fazer você não chorou"(18).

Há uma diferença nos estudos brasileiros e de outros países quanto às características das mulheres que optam pelo parto domiciliar referente à paridade. Estudos realizados nos Países Baixos (11, 13, 19-23), EUA (10, 24-26), Canadá $(5,8,9)$, Inglaterra (27) e Austrália (28-30), apontam que a maioria das mulheres que escolhe esse local é multípara, sendo que a taxa de transferência materna do domicílio para o hospital é mais alta nas primiparturientes $(6-8,10,19-21,26,27$, 29). Resultado semelhante foi encontrado em uma revisão sistemática, publicada em 2014, que analisou uma amostra de 215.257 mulheres (31).

Chama atenção que algumas pesquisas indicam maior morbidade $(8,20)$ e mortalidade materna (22), intervenções obstétricas (8, $9,19,20)$, morbidade neonatal e mortalidade perinatal $(8,27)$ nas primíparas, em comparação às multíparas assistidas no domicilio.

No Brasil, o número de primíparas que tem buscado esse tipo de assistência é cada vez mais expressivo $(32,33)$, mas ainda não existem indicadores que possibilitem avaliar os resultados obstétricos e neonatais advindos da assistência ao parto domiciliar planejado. Os dados divulgados no Sistema de Informações de Nascidos Vivos (SINASC) brasileiro abrangem os resultados de todos os partos assistidos fora do âmbito hospitalar, independente de ter sido planejado ou não.

Diante desse contexto, torna-se imprescindível conhecer as características sociodemográficas e obstétricas das mulheres que optam pelo parto domiciliar, assim como as intercorrências mais prevalentes que indicam a necessidade de transferência para o hospital, para verificar se essa modalidade de assistência é segura, independente da paridade da mulher. Assim, o objetivo desta pesquisa foi comparar os resultados obstétricos e neonatais entre primíparas e multíparas assistidas no domicílio por enfermeiras obstétricas. 


\section{MATERIAL E MÉTODO}

Trata-se de um estudo de corte transversal ${ }^{1}$ com coleta de dados retrospectiva. A amostra foi selecionada a partir dos prontuários arquivados pelas enfermeiras da Equipe Hanami - o florescer da vida - parto domiciliar planejado, que atua em Santa Catarina, Brasil, sendo incluídas as mulheres assistidas durante o trabalho de parto e/ou parto até o $10^{\circ}$ dia de puerpério, e os neonatos nascidos no domicílio, de janeiro de 2005 a dezembro de 2009.

A equipe é composta por enfermeiras obstétricas e generalistas e conta com o apoio de médicos obstetras. Somente as gestantes de baixo risco são incluídas nesse tipo de atendimento, sendo essas triadas por meio de protocolo (gestantes de baixo risco com feto único, a termo em apresentação cefálica; com até uma cesariana prévia, realizada no mínimo 2 anos antes da gestação atual). Durante o pré-natal domiciliar, também realizado pelas enfermeiras, é definida qual a instituição de saúde será a referência para transferência, caso seja necessário. No dia do parto, as enfermeiras que prestarão atendimento (três a quatro), levam todo o material, incluindo o de suporte básico de vida, para assegurar boas condições de atendimento ao binômio, nas intercorrências. Periodicamente, todas as enfermeiras obstétricas participam de treinamentos de atualização. Nos casos de transferência, é utilizado um dos automóveis das enfermeiras. O acompanhamento da puérpera e do RN ocorre no $1^{\circ}, 3^{\circ}, 4^{\circ}$ e $10^{\circ}$ dia pós-parto, ou até o $15^{\circ}$ dia, se o RN não tiver atingido o peso do nascimento (34). Esse atendimento é prestado mediante pagamento de honorários.

1 Grimes DA, Schulz KF. An overwiew of clinical research: the lay of the land. Lancet. 2002; 359(9300): 57-61.
As variáveis avaliadas neste estudo foram obtidas através dos dados mensurados e registrados pelas enfermeiras no atendimento prestado. Para a coleta de dados, utilizou-se um formulário padronizado, que foi elaborado para extrair os dados de forma sistemática, dos prontuários arquivados pela equipe. Os resultados obstétricos e neonatais foram analisados a partir das variáveis: sociodemográficas e obstétricas da gestação, obstétricas do trabalho de parto, do parto e do pós-parto e dos RNs. Foram consideradas primíparas as mulheres que estavam tendo seu primeiro parto, e multíparas, as que já haviam parido uma vez ou mais. Foram considerados os dados de trabalho de parto de todas as mulheres, e de parto e de RN, apenas das mulheres que não foram transferidas. Em virtude da necessidade de transferência de algumas mulheres durante o trabalho de parto (11), para algumas variáveis a amostra analisada é menor $(\mathrm{N}=89)$ do que a população do estudo $(\mathrm{N}=100)$.

Após preenchimento dos formulários, os dados foram digitados no programa EPI INFO - versão 2008 e analisados por estatística descritiva (frequência e percentagem). Para analisar as associações entre as variáveis foram utilizados o teste qui-quadrado, o exato de Fisher, o $t$ de Student e de Mann Whitney, de acordo com a natureza de cada variável, utilizando o software SAS versão 9.1.3. O nível de significância adotado foi de $5 \%$, portanto os valores de $p$ menores do que $0,05 \%(p$-valor $<0,05)$ apontaram resultados estatisticamente significantes. Os resultados foram discutidos com base na literatura pertinente e atualizada.

A pesquisa respeitou os procedimentos da Declaração de Helsinki e os princípios da Resolução No 196/96, e foi aprovada pelo Comitê de Ética em Pesquisa da Universidade Federal de Santa Catarina, Brasil, sob parecer No $552 / 2009$. 


\section{RESULTADOS}

Das 100 mulheres assistidas no domicílio, $73,0 \%$ eram primíparas e $27,0 \%$ multíparas. Foram transferidas para o hospital 11 mulheres ( $11,0 \%$ da amostra), sendo sete primíparas e quatro multíparas. Do total da amostra estudada, 9,0\% realizaram cesariana, sendo quatro multíparas e cinco primíparas (sem diferença estatística). Entre as multíparas, 12 eram secundigestas, quatro tercigestas, três quadrigestas ou mais, e oito tinham cesárea anterior.

A mediana de idade foi significativamente maior nas multíparas ( $\mathrm{p}=0,0019$ ). Para as demais variáveis sociodemográficas e obstétricas não houve diferença (Tabela 1).

Tabela 1. Características sociodemográficas e obstétricas das mulheres assistidas no domicílio, segundo a paridade. Santa Catarina, Brasil, 2005 a $2009(\mathrm{~N}=100)$.

\begin{tabular}{|c|c|c|c|}
\hline Variáveis & $\begin{array}{c}\text { Multípara } \\
\text { n(\%) } \\
27(27,0) \\
\end{array}$ & $\begin{array}{c}\text { Primípara } \\
\mathbf{n}(\%) \\
73(73,0) \\
\end{array}$ & Valor de $p$ \\
\hline Idade (anos) (mediana) & $30(23-43)$ & $27(21-41)$ & $0,0019^{\dagger}$ \\
\hline $20-24$ & $2(7,4)$ & $21(28,8)$ & $0,0233^{\ddagger}$ \\
\hline $25-29$ & $9(33,3)$ & $31(42,5)$ & \\
\hline $30-34$ & $8(29,6)$ & $12(16,4)$ & \\
\hline$\geq 35$ & $8(29,6)$ & $9(12,3)$ & \\
\hline Escolaridade $(n=99)$ & & & $0,3417^{\S}$ \\
\hline Médio incompleto & $1(3,8)$ & $0(0,0)$ & \\
\hline Médio completo/Superior incompleto & $11(42,3)$ & $34(46,6)$ & \\
\hline Superior completo/Pós-graduação & $14(53,8)$ & $39(53,4)$ & \\
\hline Consultas de pré-natal com o médico $(\mathrm{n}=81)$ & & & $0,7507^{\S}$ \\
\hline$<$ seis consultas & $5(23,8)$ & $11(18,3)$ & \\
\hline$\geq$ seis consultas & $16(76,2)$ & $49(81,7)$ & \\
\hline Consultas de pré-natal com enfermeiras $(n=99)$ & & & $0,2655^{\lessgtr}$ \\
\hline$<$ seis consultas & $22(84,6)$ & $63(87,5)$ & \\
\hline$\geq$ seis consultas & $2(7,7)$ & $8(11,1)$ & \\
\hline Nenhuma $^{*}$ & $2(7,7)$ & $1(1,4)$ & \\
\hline
\end{tabular}

"Encaminhadas pelo médico do pré-natal pouco tempo antes do início do trabalho de parto.

$\dagger$ Teste de Mann-Whitney. $¥$ Teste qui-quadrado. §Teste exato de Fisher.

$\mathrm{Na}$ maior parte das multíparas, o tempo transcorrido entre a primeira avaliação no domicílio e o parto foi menor do que 5 horas, sendo mais frequente do que nas primíparas $(\mathrm{p}=0,0402)$. O banho terapêutico de aspersão $(\mathrm{p}=0,0015)$, de imersão $(\mathrm{p}=0,0142)$ e o uso da bola obstétrica $(\mathrm{p}=0,0273)$ foram os métodos mais utilizados pelas primíparas para o alívio da dor (Tabela 2).
O parto em posição vertical na água foi mais escolhido pelas primíparas $(\mathrm{p}=0,0004)$. Para as demais variáveis não houve diferença estatisticamente significativa quanto à paridade (Tabela 3).

Com relação aos resultados neonatais, não houve diferença estatisticamente significativa entre primíparas e multíparas (Tabela 4). 
Tabela 2. Variáveis obstétricas do trabalho de parto das mulheres assistidas no domicílio, segundo a paridade. Santa Catarina, Brasil, 2005 a 2009 (N=100).

\begin{tabular}{|c|c|c|c|}
\hline Variáveis & $\begin{array}{c}\text { Multípara } \\
\mathbf{n}(\%) \\
27(27,0) \\
\end{array}$ & $\begin{array}{c}\text { Primípara } \\
\mathbf{n}(\%) \\
73(73,0)\end{array}$ & Valor de p \\
\hline Dilatação cervical $(\mathrm{n}=99)$ & & & $0,7233^{\S}$ \\
\hline$\leq 3 \mathrm{~cm}$ & $11(42,3)$ & $28(38,4)$ & \\
\hline$\geq 4 \mathrm{~cm}$ & $15(57,7)$ & $45(61,6)$ & \\
\hline Batimentos cardíacos fetais ( $n=99)$ & & & $0,3842^{\ddagger}$ \\
\hline Sem alteração & $25(96,2)$ & $69(94,5)$ & \\
\hline Bradicardia/taquicardia leve & $1(3,8)$ & $4(5,5)$ & \\
\hline Rotura das membranas & & & $0,4265^{\ddagger}$ \\
\hline Amniorrexe & $18(94,7)$ & $42(85,7)$ & \\
\hline Amniotomia & $1(5,3)$ & $7(14,3)$ & \\
\hline Cor do líquido amniótico $(n=62)$ & & & $1,0000^{\ddagger}$ \\
\hline Claro & $14(93,3)$ & $45(95,7)$ & \\
\hline Líquido meconial & $1(6,7)$ & $2(4,3)$ & \\
\hline Cruzamento da curva de alerta $(\mathrm{n}=97)$ & & & $0,1152^{\S}$ \\
\hline $\operatorname{Sim}$ & $6(24,0)$ & $30(41,7)$ & \\
\hline Não & $19(76,0)$ & $42(58,3)$ & \\
\hline Não foi traçado curva (fase latente) & 2 & 1 & \\
\hline Encaminhamento para hospital & & & $0,4818^{\S}$ \\
\hline Não & $23(85,2)$ & $66(90,4)$ & \\
\hline $\operatorname{Sim}$ & $4(14,8)$ & $7(9,6)$ & \\
\hline Tempo dilatação/expulsão (n=89) & & & $0,0402^{\ddagger}$ \\
\hline $5 \mathrm{~min}$ a $4: 59 \mathrm{~min}$ & $16(69,6)$ & $25(37,9)$ & \\
\hline 5 horas a 9:59 min & $3(13,0)$ & $24(36,4)$ & \\
\hline 10 horas a $14: 59 \mathrm{~min}$ & $4(17,4)$ & $12(18,2)$ & \\
\hline 15 horas a $19: 59 \mathrm{~min}$ & $0(0,0)$ & $5(7,6)$ & \\
\hline \multicolumn{4}{|l|}{ Distócia funcional $^{*}(\mathbf{n}=99)$} \\
\hline Ausente & $18(66,7)$ & $52(71,2)$ & $0,6582 \S$ \\
\hline Bradissistolia/hipossistolia & $8(29,6)$ & $20(27,4)$ & $0,8253 \S$ \\
\hline Taquissistolia & $0(0,0)$ & $1(1,4)$ & $1,0000 \ddagger$ \\
\hline \multicolumn{4}{|l|}{ Métodos não-farmacológicos $\dagger$} \\
\hline Banho de aspersão & $9(33,3)$ & $50(68,5)$ & $0,0015 \S$ \\
\hline Banho de imersão & $10(37,0)$ & $47(64,4)$ & $0,0142 \S$ \\
\hline Massagem lombo-sacra & $16(59,3)$ & $37(50,7)$ & $0,4456 \S$ \\
\hline Bola & $6(22,2)$ & $34(46,6)$ & $0,0273 \$$ \\
\hline
\end{tabular}

*Oito mulheres apresentaram duas distocias. ${ }^{\dagger}$ Cada mulher recebeu mais de um método.

‡ Teste exato de Fisher. \$Teste qui-quadrado. 
Tabela 3. Variáveis do parto e do pós-parto imediato das mulheres assistidas no domicílio, segundo a paridade. Santa Catarina, Brasil, 2005 a $2009(\mathrm{~N}=89)$.

\begin{tabular}{|c|c|c|c|}
\hline Variáveis & $\begin{array}{c}\text { Multípara } \\
\text { n(\%) } \\
23(25,8)\end{array}$ & $\begin{array}{c}\text { Primípara } \\
\mathrm{n}(\%) \\
66(74,2)\end{array}$ & Valor de $\mathrm{p}$ \\
\hline Posição de parto & & & $0,0004^{\dagger}$ \\
\hline Vertical na água & $10(43,5)$ & $54(81,8)$ & \\
\hline Vertical/sims fora da água & $13(56,5)$ & $12(18,2)$ & \\
\hline Rotura das membranas & & & $1,0000^{\dagger}$ \\
\hline Amniorrexe & $6(100,0)$ & $19(95,0)$ & \\
\hline Amniotomia & $0(0,0)$ & $1(5,0)$ & \\
\hline Cor do líquido amniótico & & & $0,2184^{\dagger}$ \\
\hline Claro & $4(66,7)$ & $18(90,0)$ & \\
\hline Líquido meconial & $2(33,3)$ & $2(10,0)$ & \\
\hline Condições do períneo $(\mathrm{n}=87)$ & & & $0,4303^{\dagger}$ \\
\hline Sem trauma ou sem sutura & $9(39,1)$ & $35(54,7)$ & \\
\hline Laceração de $1^{\circ}$ grau com sutura & $14(60,9)$ & $28(43,8)$ & \\
\hline Episiotomia & $0(0,0)$ & $1(1,6)$ & \\
\hline \multicolumn{4}{|l|}{ Contato pele a pele } \\
\hline $\operatorname{Sim}$ & $23(100,0)$ & $66(100,0)$ & \\
\hline Amamentação na $1^{\mathrm{a}}$ hora & & & $0,0689^{\ddagger}$ \\
\hline Sucção efetiva & $20(87,0)$ & $43(67,2)$ & \\
\hline \multicolumn{4}{|l|}{ Intercorrência no secundamento } \\
\hline Dequitação prolongada & $12(44,4)$ & $33(50,0)$ & $0,9459^{\ddagger}$ \\
\hline Nenhuma & $10(37,0)$ & $27(40,9)$ & $0,9963^{\ddagger}$ \\
\hline Hipotensão & $1(3,7)$ & $5(7,6)$ & $1,0000^{\dagger}$ \\
\hline Hemorragia & $1(3,7)$ & $4(6,1)$ & $1,0000^{\dagger}$ \\
\hline
\end{tabular}

*Nove mulheres tiveram mais de uma intercorrência.

$†$ Teste exato de Fisher. $¥$ Teste de qui-quadrado.

Tabela 4. Variáveis dos recém-nascidos assistidos no domicílio, segundo paridade. Santa Catarina, Brasil, 2005 a $2009(\mathrm{~N}=89)$.

\begin{tabular}{|c|c|c|c|}
\hline Variáveis & $\begin{array}{c}\text { Multípara } \\
\text { n(\%) } \\
23(25,8)\end{array}$ & $\begin{array}{c}\text { Primípara } \\
n(\%) \\
66(74,2)\end{array}$ & Valor de $\mathrm{p}$ \\
\hline Apgar do $1^{\circ}$ minuto & & & $0,5656^{\ddagger}$ \\
\hline$<7$ & $0(0,0)$ & $3(4,5)$ & \\
\hline$\geq 7$ & $23(100,0)$ & $63(95,5)$ & \\
\hline Apgar do $5^{\circ}$ minuto & & & $1,0000^{\ddagger}$ \\
\hline$<7$ & $0(0,0)$ & $1(1,5)$ & \\
\hline$\geq 7$ & $23(100,0)$ & $65(98,5)$ & \\
\hline Classificação do recém-nascido & & & $0,7606^{\ddagger}$ \\
\hline Pequeno para a idade gestacional & $0(0,0)$ & $1(1,5)$ & \\
\hline Adequado para a idade gestacional & $22(95,7)$ & $59(89,4)$ & \\
\hline Grande para a idade gestacional & $1(4,3)$ & $6(9,1)$ & \\
\hline
\end{tabular}


Continuação Tabela 4.

\begin{tabular}{lccc}
\hline Intercorrência até $\mathbf{o} \mathbf{1 5}^{\mathbf{0}} \mathbf{d i a}^{*}(\mathbf{n}=\mathbf{8 8})$ & $12(44,4)$ & $27(37,0)$ & $0,4972^{\S}$ \\
Icterícia pós-parto & $7(25,9)$ & $24(32,9)$ & $0,5046^{\S}$ \\
Nenhuma & $6(22,2)$ & $14(19,2)$ & $0,7355^{\S}$ \\
Peso $<$ do que esperado no $10^{\circ}$ dia & $0(0,0)$ & $2(2,7)$ & $1,0000^{\ddagger}$ \\
Peso $<$ do que esperado no $15^{\circ}$ dia
\end{tabular}

${ }^{*}$ Um recém-nascido de primípara foi transferido na primeira hora de vida, três tiveram duas intercorrências.

${ }^{\dagger}$ Teste $t$ de Student. ${ }^{\ddagger}$ Teste exato de Fisher. ${ }^{\circledR}$ Teste qui-quadrado.

\section{DISCUSSÃO E CONCLUSÕES}

Os resultados obstétricos e neonatais foram satisfatórios nos dois grupos analisados (primíparas e multíparas), sendo que a taxa de transferência para o hospital foi baixa em ambos. A necessidade de transferência foi similar nesses grupos, diferente dos estudos realizados em outros países, nos quais essa taxa é maior nas primíparas $(6-8,10,19-21,26$, $27,29)$. Revisão sistemática acerca dessa modalidade de atendimento encontrou resultado similar, sendo que a taxa de transferência das nulíparas oscilou entre 23,4 a $45,4 \%$ e das multíparas entre 5,8 a 12,0\% (31).

Os achados encontrados foram semelhantes aos de pesquisas realizadas em países onde o parto é assistido no domicílio pelas midwives e é respeitado e reconhecido pelo sistema de saúde. Entretanto, chama atenção o maior número de primíparas que optam por esse local na realidade brasileira, conforme já apontado em outra investigação (32), uma vez que em estudos realizados nos Países Baixos (11, 13, 19-23), EUA (10, 24-26), Canadá $(5,8,9)$, Austrália (28-30) e Inglaterra $(27,35)$, esse grupo de mulheres tende a preferir a assistência hospitalar. Em pesquisa realizada nos Países Baixos, quase três vezes mais multíparas optaram pelo parto domiciliar em relação às primíparas (20). No Brasil, as mulheres podem estar optando pelo parto no domicílio na tentativar de evitar a violência obstétrica $(14,16,18)$, poder ter suas crenças e opções respeitadas $(14,16)$ e evitar intervenções desnecessárias $(14,16)$.

$\mathrm{O}$ ambiente domiciliar favoreceu a livre escolha da mulher sobre as posições escolhidas no parto e em especial as primíparas, que mais optaram pelo parto vertical na água. Esse resultado é similar a estudos internacionais que apontam que as mulheres assistidas em casa optam por posições não litotômicas e pelo parto na água $(6,28)$, embora não tenham analisado se houve diferença dessa variável em relação à paridade. A liberdade para parir em posição mais confortável pode ser um dos fatores que levaram as mulheres a optar pelo parto domiciliar, já que a posição de litotomia é amplamente utilizada em hospitais brasileiros $(17,36)$ e a possibilidade de parir na parto na água é pouco oferecida nos serviços públicos de saúde.

A maioria das variáveis estudadas não apresentou diferença estatisticamente significativa. As variáveis que apresentaram diferença foram: a idade, o tempo transcorrido entre a primeira avaliação e a expulsão, o uso de alguns métodos não-farmacológicos para o alívio da dor e a posição de parto.

As mulheres estavam na faixa etária de baixo risco reprodutivo, sendo que as multíparas estavam numa faixa etária maior do que as primíparas, como esperado, possuíam elevado grau de escolaridade e uma fonte de renda. Tais dados são semelhantes a outra pesquisa realizada no Brasil (32) e em outros países $(5,6,9-11,13,20,24-26,28,30)$. Essas características sociodemográficas podem ter facilitado a escolha, uma vez que, atualmente, no Brasil, o atendimento ao parto domici- 
liar exige um planejamento financeiro.

As multíparas apresentaram menor tempo transcorrido entre o início do atendimento domiciliar até a expulsão, resultado também esperado, uma vez que essas têm períodos clínicos de dilatação e de expulsão menores em relação às primíparas.

Neste estudo, a porcentagem de mulheres que tiveram tempo superior a 10 horas de trabalho de parto pode ser decorrente do elevado número de mulheres que se encontrava na fase latente do trabalho de parto no início do acompanhamento ou também decorrente do respeito à evolução fisiológica do processo de parir, enquanto parâmetros maternos e fetais estivessem normais. Assim, a ocorrência de bradissistolia e hipossistolia também pode ter contribuído para esse resultado e para o cruzamento da curva de alerta em $36,0 \%$ dos casos. Entretanto, vale a pena destacar que o tempo maior do que 15 horas de acompanhamento foi observado somente entre as primiparturientes.

É notório esperar que o ambiente escolhido para o processo parturitivo possa influenciar no tempo de trabalho de parto, uma vez que, no hospital, intervenções no intuito de acelerar o trabalho de parto, como a indução e a condução do trabalho de parto, assim como a amniotomia são práticas mais frequentes $(5,9,12,19,24)$.

As enfermeiras disponibilizaram uma grande diversidade de métodos não-farmacológicos para alívio da dor. As primíparas fizeram mais uso de alguns métodos, com diferença estatisticamente significativa, demonstrando que essas necessitaram de maior suporte para a diminuição da sensação dolorosa. Estudos realizados nos Países Baixos (19, 20) e no Canadá (8) mostraram que as primíparas utilizam mais medicação para alívio da dor $(34,1$ e $41,1 \%)$ quando comparadas com as multíparas (15,5 e 7,7\%). Existem poucas informações sobre o uso desses métodos no domicílio. Em pesquisa realizada na Suíça (6), os métodos mais utilizados foram a movimentação e a massagem, contudo, não foi analisada a diferença com relação à paridade. O banho (aspersão e imersão), a massagem e a bola foram os métodos mais escolhidos. O que leva uma parturiente a preferir um ou outro método ainda não está bem esclarecido pelas pesquisas, sendo que é importante investigar essa temática, assim como avaliar se o maior tempo de trabalho de parto é uma variável confundidora que está associada com a maior utilização dos métodos não-farmacológicos pelas primíparas.

A laceração perineal é um desfecho que apresenta diferença quando se compara a paridade das mulheres, acometendo de 61,3 a $85,73 \%$ das primíparas e 26,9 a $52,0 \%$ (8, $19,35)$ das multíparas. No presente estudo, embora a laceração perineal tenha acometido também mais as multíparas $(60,9 \%)$ do que as primíparas $(43,8 \%)$, essa variavel não apresentou diferença estatisticamente significativa. Cabe ressaltar que metade das mulheres, 44, independente da paridade, não necessitou de sutura, e apesar de haver um maior número de lacerações perineais do que as descritas na literatura $(6,9,26,32)$, essas limitaram-se ao $1^{\circ}$ grau, não havendo nenhuma laceração de $2^{\circ}, 3^{\circ}$ ou $4^{\circ}$ grau. Esses resultados diferem de outras pesquisas em que a taxa de laceração de $2^{\circ}$ grau foi de $36,4 \%$ (22) e de $3^{\circ}$ e/ou $4^{\circ}$ grau variou entre 0,4 a $1,2 \%(9,26,30)$. Em estudo que analisou essa intercorrência em relação à paridade, encontrou-se taxa de laceração de $2^{\circ}$ grau de $29,0 \%$ nas nulíparas e de $20,3 \%$ nas multíparas, e de $3^{\circ}$ grau de $7,1 \%$ nas nulíparas e de $0 \%$ nas multíparas (35).

Corroborando com os dados de outras pesquisas realizadas nos Estados Unidos (10, 26), Canadá $(5,8,9)$, Austrália $(29,34)$ e Inglaterra (35), em que a taxa de episiotomia oscilou entre 0,4 a $4,3 \%$, a taxa da presente investigação foi semelhante $(1,1 \%)$, sendo utilizada em apenas uma primípara. Essa baixa prevalência, neste estudo pode ter sido decorrente da própria assistência, baseada em evidência científica prestada pelas enfermeiras. Entretanto, em estudos desen- 
volvidos nos Países Baixos, essa taxa é mais elevada $(7,19)$, inclusive atingindo 52,4\% em primíparas e somente $15,8 \%$ das multíparas (19). Cabe destacar que a episiotomia é amplamente utilizada nos hospitais brasileiros, chegando a ser realizada em 53,5\% das parturientes submetidas ao parto normal, segundo dados do inquérito nacional sobre nascimento e parto, realizado no Brasil em 2011 e 2012 (17).

Quanto ao rompimento artificial das membranas, a literatura internacional (20) aponta pouca diferença entre primíparas $(19,6 \%)$ e multíparas $(21,4 \%)$. No presente estudo foi predominante a preservação da integridade das membranas, o que pode ter favorecido o bem-estar fetal e a permanência do líquido amniótico claro. Esses achados são similares aos dos estudos internacionais, nos quais a taxa de amniotomia oscila entre 5,0 a $22,4 \%(5,8-10,20)$. No entanto, são expressivamente menores do que as taxas encontradas em hospitais e centros de parto normal brasileiros $(17,36,37)$.

A ocorrência de líquido meconial foi inexpressiva, independente da paridade, sendo similar a outros estudos, em que variou entre 2,3 a $13,4 \%(5,19,24)$; resultado que pode ter influenciado na elevada frequência de escore de Apgar $\geq 7$ e apenas uma transferência de RN para UTIN, sendo essa não relacionada com a assistência prestada. A boa vitalidade dos RNs provavelmente foi devido à evolução fisiológica do parto e também por se tratar de mulheres de baixo risco.

Os resultados da presente investigação também mostraram a adoção de boas práticas como o contato pele a pele da mãe com o RN em todos os partos e a alta taxa de amamentação na primeira hora de vida. O conhecimento das mulheres sobre o benefício dessas práticas e a filosofia de assistência da equipe pode ter contribuído para esses resultados. Dados similares foram constatados em estudo suíço (6). No entanto, estudo realizado em ambiente hospitalar apontou que, embora $78,3 \%$ das mulheres tocaram o RN na sala de parto, somente 33,0\% levaram-no ao seio (36).

Quanto aos resultados neonatais, não houve diferença significativa em relação à paridade, sendo que os escores de Apgar de $1^{\circ}$ e $5^{\circ}$ minuto foram elevados. Esses achados são semelhantes a outros estudos $(5,10,24$, 28, 30, 32). Entretanto, chama atenção que um estudo que comparou resultados neonatais de acordo com a paridade da mulher, encontrou melhores resultados de Apgar do $5^{\circ}$ minuto e menor taxa de internação em UTIN nas multíparas (19).

A baixa transferência de RN é congruente com as pesquisas na qual a necessidade de transferência foi de 1,0\% das mulheres que pariram em casa (26).

Quanto às intercorrências que acometeram as mulheres no pós-parto imediato, ocorreu uma pequena porcentagem de hemorragia pós-parto; um resultado congruente ao descrito em outras investigações $(5,6$, $9,12,19,30,38)$, sendo mais frequente em primíparas do que em multíparas, entretanto sem diferença estatisticamente significante. No estudo de Jonge et al., 2013, a hemorragia acometeu apenas 2,9\% das mulheres que pariram em casa e 3,9\% das mulheres que pariram no hospital (13).

Não houve nenhum caso de transferência da mulher após o parto. Nas intercorrências de pós-parto imediato, as medidas necessárias foram tomadas e houve estabilização do quadro, o que diverge de algumas pesquisas que apresentam taxa de transferência nesse período de $1,7 \%$, independente da paridade (26), de 1,6 a 8,9\% nas nulíparas e de 1,6 a $5,5 \%$ nas multíparas (31).

Diferente de outros países, no Brasil, a maioria das mulheres assistidas no domicílio é primípara. Os dados analisados indicam bons resultados maternos e neonatais, tanto em primíparas quanto em multíparas, e são semelhantes aos de outros países onde essa prática é frequente e integrada ao sistema de saúde. Não há diferença significativa na taxa de transferência para o hospital, quando ana- 
lisada a paridade.

Entre as limitações do estudo estão a coleta retrospectiva dos dados e a amostra relativamente pequena, com um potencial viés, uma vez que são partos de mulheres que desejam uma assistência não intervencionista e, acima de tudo, acreditam no seu poder parturitivo.

A existência de poucos estudos sobre esse tipo de assistência no Brasil não permitiu a discussão das variáveis com indicadores desse país. Dessa forma, a maioria dos resultados foi comparada com pesquisas realizadas em outros países, sendo que a análise dos resultados de acordo com a paridade é escassa, o que evidencia a necessidade de outras investigações. Conhecer as características da população atendida, o comportamento das principais variáveis de desfecho, além do tipo e prevalência de complicações, pode contribuir para a melhor preparação dos profissionais, assim como para a revisão e melhoria dos protocolos de atendimento ao parto domiciliar planejado, no que tange à segurança materna e neonatal.

Poucas variáveis apresentaram diferença estatisticamente significativa entre os grupos estudados, como maior idade entre as multíparas, menor tempo transcorrido entre a primeira avaliação e a expulsão, assim como a menor necessidade de uso de alguns métodos não-farmacológicos e o parto fora da água pelas multíparas.

Apesar das limitações, o estudo contribui para fomentar a avaliação da assistência ao parto domiciliar planejado no Brasil e, além disso, divulga esse tipo de assistência e propicia maior visibilidade à atuação autônoma das enfermeiras obstétricas, que ainda é insipiente neste país.

As complicações e distócias ocorridas na amostra, apesar de pouco frequentes, foram prontamente solucionadas pela equipe e as transferências necessárias foram realizadas sem intercorrências, sendo que os resultados após as transferências mostram-se positivos para o binômio; fatos observados tanto em primíparas quanto em multíparas.

Os achados contribuem para a construção do conhecimento acerca da atuação da enfermeira obstétrica na atenção ao parto domiciliar e indicam a necessidade de outras investigações sobre essa prática no cenário nacional. Os bons resultados deste estudo indicam que o domicílio pode ser um bom local para o parto independente da paridade da mulher, desde que seguido um protocolo rigoroso de triagem de atendimento.

\section{REFERÊNCIAS}

1. Organização Mundial da Saúde. Assistência ao parto normal: um guia prático. Genebra: OMS; 1996.

2. Lei $N^{\circ} .7498$ de 25 de junho de 1986 . Dispõe sobre a regulamentação do exercício da enfermagem, e dá outras providências. Conselho Regional de EnfermagemSC.7.ed. Florianópolis: COREN; 2008. p. 53.

3. Agência Nacional de Saúde Complementar. O modelo de atenção obstétrica no setor de saúde suplementar no Brasil: cenários e perspectivas. Rio de Janeiro: ANS; 2008.

4. Committee opinion. Planned home birth. Obstetrics \& Gynecology. 2011; 117(2, part 1): 425-8.

5. Janssen PA, Lee SK, Ryan EM, Etches DJ, Farquharson DF, Peacok D, et al. Outcomes of planned home births versus planned hospital births after regulation of Midwifery in British Columbia. CMAJ. 2002; 166(3): 315-23.

6. Ackermann-Leibrich U, Voegeli T, Gunter-Witt K, Kunz I, Zullig M, Schindler C, et al. Home versus hospital deliveries: follow up study of matched pairs for procedures and outcomes. BMJ. 1996; 313: 1313-8.

7. Amerlink-Verburg MP, Verloove-Vanhorick SP, Hakkenberg RMA, Veldhui- 
jen IME, Gravenhorst JB, Buitendijk SE. Evaluation of 280,000 cases in Dutch midwifery practices: a descriptive study. BJOG. 2008; 115: 570-8.

8. Hutton EK, Reitsma AH, Kaufman K. Outcomes associated with planned home and planned hospital births in low-risk women attended by midwives in Ontario, Canadá, 2003-2006: a retrospective cohort study. Birth. 2009; 36(3): 180-9.

9. Janssen PA, Saxel L, Page L, Klein MC, Liston RM, Lee SK. Outcomes of planned home birth with registered midwife versus planned hospital birth with midwife or physician. CMAJ. 2009; 181(6-7): 377-83. doi:10.1503/cmaj.081869.

10. Johnson KC, Daviss BA. Outcomes of planned home births with certifed professional midwives: large prospective study in North America. BMJ on line. $2005 ; 330$.

11. Jonge A, Goes VD, Ravelli ACJ, AmelinkVerburg MP, Mol BW, Nijhuis JG, et al. Perinatal mortality and morbidity in a nationalwide cohort of 529688 low risk planned home and hospital births. BJOG. 2009; 116(9): 1177-84.

12. Woodcock HC, Read AW, Bower C, Stanley FJ, Moore DJ. A matched cohort study of planned home and hospital births in Western Australia 1981-1987. Midwifery. 1994; 10(3): 125-35.

13. Jonge A, Mesman JAJM, Mannien J, Zwart J. Severe adverse maternal outcomes among low risk women with planned home versus hospital births in the Netherlands: nationwide cohort study. BMJ. 2013; 346: f3263-73.

14. Feyer ISS, Monticelli M, Volkmer C, Burigo RA. Publicações científicas brasileiras de enfermeiras obstétricas sobre o parto domiciliar: revisão sistemática de literatura. Texto contexto-enferm. 2013; 22(1): 247-56.

15. Kruno RB, Bonilha ALL. Parto no domicílio na voz das mulheres: uma perspectiva à luz da humanização. Rev Gaucha
Enferm. 2004; 25(3): 396-407.

16. Medeiros RMK, Santos IMM, Silva LR. A escolha pelo parto domiciliar: história de vida de mulheres que vivenciaram esta experiência. Esc. Anna Nery. 2008; 12(4): 765-72.

17. Leal MC, Pereira APE, Domingues RMSM, Filha MMT, Dias MAB, Nakamura-Pereira $\mathrm{M}$ et al. Intervenções obstétricas durante o trabalho de parto e parto em mulheres brasileiras de risco habitual. Cad Saude Publica. 2014; 30, sup: S17-S47.

18. Núcleo de Opinião Pública da Fundação Perseu Abramo. Mulheres brasileiras e gênero nos espaços público e privado. Pesquisa de opinião pública. 2010.

19. Wiegers TA, Keirse MJNC, Zee JVD, Berghs GAH. Outcome of planned home and planned hospital births in low risk pregnancies: prospective study in midwifery practices in the Netherlands. BMJ. 1996; 313: 1309-13.

20 . Wiegers $\mathrm{T}$. The quality of maternity care services as experiences by women in the Netherlands. BMC Pregnancy Childbirth. 2009; 9(18).

21. Anthony S, Buitendijk SE, Offerhaus PM, Van Dommelen P, Van der Pal-de Bruin KM. Maternal factors and the probability of a planned home birth. BJOG. 2005; 112: 748-753.

22. Van der Kooy J, Poeran J, Graaf JP, Birnie E, Denktas S, Steegers EAP, Bonsel G. Planned home compared with planned hospital births in The Netherlands. Intrapartum and early neonatal death in low-risk pregnancies. Obstetrics \& gynecology. 2011; 118: 1037-46.

23. Li Y, Townend J, Rowe R, Knight M, Brocklehurst P, Hollowell J. The effect of maternal age and planned place of birth on intrapartum outcomes in healthy women with straightforward pregnancies: secondary analysis of the Birthplace national prospective cohort study. BMJ Open. 2014; 4: e004026. 
24. Wax JR, Pinette MG, Cartin A, Blackstone J. Maternal and newborn morbidity by birth facility among selected United States 2006 low-risk births. Am J Obstet Gynecol. 2010; 152: e1-e5.

25. Cheng YW, Snowden JM, King TL, Caughey AB. Selected perinatal outcomes associated wit planned home births in the United States. Am J Obstet Gynecol. 2013; 209: 325.e1-8.

26. Cheyney M, Bovbjerg M, Everson C, Gordon W, Hannibal D, Vedam S. Outcomes of care for 16,924 Planned Home Births in the United States: The Midwives Alliance of North America Statistics Project, 2004 to 2009. J Midwifery Womens Health. 2014; 59(1): 17-27.

27. Birthplace in England Collaborative Group. Perinatal and maternal outcomes by planned place of birth for healthy women with low risk pregnancies: the Birthplace in England national prospective cohort study. BMJ. 2011, 343.

28. Mcmurtrie J, Catling-Paul C, Teate A, Caplice S, Chapman M, Homer C. The St. George homebirth program: an evaluation of the first 100 booked women. Aust N Z J Obstet Gynecol. 2009; 49(6): 631-6.

29. Homer CSE, Thornton C, Scarf VL, Ellwood DA, Oats JJN, Foureur MJ et al. Birthplace in New South Wales, Australia: an analysis of perineal outcomes using routinely collected data. BMC Pregnancy Childbirth. 2014; 14: 206.

30. Kennare RM, Keirse MJNC, Tucker GR, Chan AC. Planned home and hospital births in South Australia, 1991-2006: differences in outcomes. Med J Aust. 2009; 192(2): 76-80.

31. Blix E, Kumie M, Kjaergaard H, Oian P,
Lindgren $\mathrm{H}$. Transfer to hospital in planned home births: a systematic review. BMC Pregnancy Childbirth. 2014; 14: 179.

32. Colacioppo PM, Koiffman MD, Riesco MLG, Schneck CA, Osava RH. Parto domiciliar planejado: resultados maternos e neonatais. Rev de Enfermagem Referência. 2010; 2: 81-90.

33. Koettker JG, Bruggemann OM, Dufloth RM, Knobel R, Monticelli M. Resultado de partos domiciliares atendido por enfermeiras de 2005 a 2009 em Florianópolis, SC. Rev Saude Publica. 2012; 46(4): 747-50.

34. Feyer ISS, Silva J, Koettker JG, Calvette MF, Burigo RA, Collaço VS. O florescer da vida: parto domiciliar planejado, orientações para gestação, parto e pósparto. Florianópolis: Lagoa; 2009.

35. Smith L, Price N, Simonite V, Burns. Incidence of and risk factors for perineal trauma: a prospective observational study. BMC Pregnancy Childbirth. 2013; $13(59)$.

36. D'Orsi E, Chor D, Giffin K, Ângulo-Tuesta A, Barbosa GP, Gama AS, et al. Qualidade da atenção ao parto em maternidades do Rio de Janeiro. Rev Saude Publica. 2005; 34(4): 646-54.

37. Koiffman MD, Schneck CA, Reisco MLG, Bonadio IC. Risk factors for neonatal transfers from the Sapopemba FREEstanding birth centre to a hospital in São Paulo, Brazil. Midwifery. 2009.

38. Nove A, Berrington A, Matthews Z. Comparing the odds of postpartum haemorrhage in planned home birth against planned hospital birth: results of an observational study of over 500,000 maternities in the UK. BMC Pregnancy Childbirth. 2012; 12(130). 